\section{Programa de Triagem Neonatal para hipotireoidismo congênito de Santa Catarina, Brasil: avaliação etiológica no primeiro atendimento}

\author{
Newborn Screening Program for congenital hypothyroidism of the \\ State of Santa Catarina, Brazil: etiological investigation in the first visit
}

Marilza Leal Nascimento', Fernanda Hostim Rabello²,

Masanao Ohira ${ }^{3}$, Genoir Simoni ${ }^{4}$, Edson Cechinel',

Rose Marie Muller Linhares ${ }^{4}$, Paulo César Alves da Silva ${ }^{4}$

\section{RESUMO}

Objetivo: Avaliar a etiologia, no primeiro atendimento, dos casos de hipotireoidismo congênito primário (HCP) identificados pelo Programa de Triagem Neonatal de Santa Catarina entre julho de 2007 e junho de 2009. Sujeitos e métodos: Estudo prospectivo com 45 pacientes com HCP confirmado. Para o diagnóstico etiológico, eram realizados na primeira consulta: anamnese, exames físico e complementares (TSH, tiroxina livre, tireoglobulina, idade óssea, ultrassonografia de tireoide). Resultados: Estabeleceu-se o diagnóstico etiológico na primeira consulta em $53,33 \%$. Disgenesia representou $51,11 \%$, sendo $20 \%$ hipoplasia, $13,3 \%$ atireose e $17,7 \%$ ectopia; e 2,2\% foram diagnosticados com disormoniogênese. Hérnia umbilical foi o sinal mais prevalente $(48,89 \%)$ e $20 \%$ não apresentaram manifestação clínica. Aqueles com disgenesia apresentaram diferença significativa $(p<0,05)$ pela via de parto cesária, idade óssea atrasada eTSH sérico muito elevado. Conclusões: $\mathrm{A}$ abordagem diagnóstica realizada no primeiro atendimento determina a etiologia do HCP em $53,3 \%$ dos casos. A metade dos pacientes apresenta disgenesia tireoidiana. Arq Bras Endocrinol Metab. 2012;56(9):627-32

Descritores

Hipotireoidismo congênito; triagem neonatal; glândula tireoide; ultrassonografia

\begin{abstract}
Objective: To evaluate the etiology of primary congenital hypothyroidism (PCH) identified in the Newborn Screening Program from the state of Santa Catarina, Brazil, from July 2007 to June 2009 in the first visit. Subjects and methods: A prospective study was performed in 45 patients with $\mathrm{PCH}$. For the etiological diagnosis, history, physical examination, and additional tests (TSH, free thyroxine, thyroglobulin, bone age assessment, thyroid ultrasound) were carried out in the first visit. Results: The etiology was established in the first visit in $53.3 \%$ of cases. Thyroid dysgenesis represented $51.11 \%$ of the cases, from which $20 \%$ showed hypoplastic thyroid, $13.3 \%$ showed athyreosis, and $17.7 \%$ showed ectopic glands; $2.2 \%$ were diagnosed with dyshormonogenesis. Umbilical hernia was the most prevalent sign (48.89\%) and $20 \%$ had no clinical manifestations. Patients with dysgenesis showed significant differences $(p<0.05)$ in terms of cesarean section delivery, delayed bone age, and very high serum TSH. Conclusions: The diagnostic approach used at first visit for $\mathrm{PCH}$ patients may determine the etiology in $53.3 \%$ of cases. Half of patients had thyroid dysgenesis. Arq Bras Endocrinol Metab. 2012;56(9):627-32
\end{abstract}

\section{Keywords}

Congenital hypothyroidism; neonatal screening; thyroid gland; ultrasonography
${ }^{1}$ Hospital Infantil Joana de Gusmão, Serviço de Endocrinologia Pediátrica; Universidade Federal de Santa Catarina (UFSC), Departamento de Pediatria, Florianópolis, SC, Brasil 2 UFSC, Florianópolis, SC, Brasil ${ }^{3}$ UFSC, Departamento de Informática e Estatística, Florianópolis, SC, Brasil ${ }^{4}$ Hospital Infantil Joana de Gusmão, Serviço de Endocrinologia Pediátrica, Florianópolis, SC, Brasil

Correspondência para: Marilza Leal Nascimento Rua Rui Barbosa, 152 - Agronômica 88025-301 - Florianópolis, SC, Brasi marilzaleal@brturbo.com

Recebido em 10/Maio/2012 Aceito em 27/Ago/2012 


\section{INTRODUÇÃO}

$\mathrm{O}$

hipotireoidismo congênito (HC) é detectado em

$1: 3.000$ a 4.000 nascidos vivos e afeta duas vezes mais o sexo feminino (1-6). É a causa mais comum de deficiência mental passível de prevenção $(2,4)$. Estabelecer o diagnóstico e iniciar o tratamento precocemente é fundamental para prevenir sequelas $(6,7)$.

O diagnóstico clínico do HC é dificultado pela ausência de sinais específicos (8) e porque grande parcela dos pacientes acometidos apresenta-se normal ao nascimento.

A fim de facilitar o diagnóstico e evitar danos do hipotireoidismo não tratado, foram criados programas de deteç̧ão precoce, conhecidos como "teste do pezinho" ou triagem neonatal.

Em Santa Catarina, o Programa Estadual de Triagem Neonatal (PTN-SES/SC) foi implantado em julho de 1992 e faz a triagem para HC por meio da dosagem de hormônio tireoestimulante (TSH). Níveis de TSH elevado e tiroxina (T4) reduzido fazem o diagnóstico de HC.

O HC pode ser classificado de acordo com o nível da lesão, primário (HCP) quando a alteração ocorre na glândula tireoide, secundário na hipófise e terciário no hipotálamo (9). Esses dois últimos são raros.

O HCP em $80 \%$ dos casos se deve à disgenesia da tireoide, que abrange ausência da glândula (atireose), localização anormal (ectopia) e redução do tecido tireoidiano (hipoplasia). Em 10\% dos casos de HCP, ocorre disormoniogênese, alteração na síntese dos hormônios tireoidianos $(1,10-12)$.

O HCP pode ser transitório em $5 \%$ a $10 \%$ dos casos e decorre da passagem transplacentária de anticorpos tireoidianos maternos, ou por uso materno de drogas ou substâncias antitireoidianas, exposição materna ou neonatal a agentes iodados ou por deficiência na ingestão de iodo (4).

A etiologia do HCP pode exercer um papel fundamental para determinar a gravidade no momento do diagnóstico, como também suas consequências $(6,7)$. A definição da etiologia do HCP pode ser feita pela dosagem de tireoglobulina e pela realização de cintilografia tireoidiana com tecnésio 99 ou iodo 123 e/ou pela ultrassonografia - método de escolha para investigação etiológica $(6,13,14)$. A associação do nível da tireoglobulina e ultrassonografia pode distinguir entre atireose e ectopia glandular.

Os Serviços de Referência em Triagem Neonatal brasileiros realizam investigação etiológica somente após os 3 anos de idade, devido à dificuldade de realizar exames complementares de imagem no primeiro aten- dimento. A partir de 2007, foi implementada, no Serviço de Referência em Triagem Neonatal (SRTN) de Santa Catarina, a avaliação etiológica no primeiro atendimento, que consiste em realizar dosagem de TSH, T4 livre, tireoglobulina, ultrassonografia de tireoide e radiografia dos joelhos para cálculo da idade óssea.

O presente estudo tem com objetivo avaliar, no primeiro atendimento, a etiologia dos casos de hipotireoidismo congênito diagnosticados pelo PTN-SES/SC no período de julho de 2007 a junho de 2009 , fornecendo subsídios para aprimorar a abordagem diagnóstica e terapêutica dos pacientes com HCP e possibilitar um precoce aconselhamento genético das famílias.

\section{SUJEITOS E MÉTODOS}

Todas as crianças triadas pelo Programa de Triagem Neonatal de Santa Catarina (PTN-SC) entre 2007 e 2009 e convocadas para primeira consulta por TSH neonatal alterado foram atendidas no Ambulatório de Endocrinologia Pediátrica do Hospital Infantil Joana de Gusmão (HIJG) da Secretaria de Estado de Saúde de Santa Catarina, localizado na cidade de Florianópolis (Serviço de Referência em Triagem Neonatal para o estado de Santa Catarina).

No período estudado, o PTN-SC utilizou, para o diagnóstico de HCP, a análise de TSH em papel-filtro, com coleta realizada idealmente entre o terceiro e quinto dia de vida e valor de referência de $10 \mathrm{mUI} / \mathrm{L}$. As crianças com TSH maior que $20 \mathrm{mUI} / \mathrm{L}$ foram convocadas em caráter de urgência para consulta médica. Aquelas que apresentaram resultados limítrofes (entre 10 e $20 \mathrm{mUI} / \mathrm{L}$ ) foram submetidas à nova coleta em papel-filtro e convocadas caso o resultado confirmasse um TSH maior que $9 \mathrm{mUI} / \mathrm{L}$.

Durante a primeira consulta, após anamnese detalhada (história familiar e pré-natal), as crianças foram examinadas por um dos cinco médicos da equipe. Os seguintes sinais e sintomas foram sistematicamente pesquisados: choro rouco, hérnia umbilical, fontanela posterior ampla, hipotonia, pele fria, livedo reticular, base nasal alargada, constipação, dificuldade às mamadas, macroglossia, icterícia prolongada (mais que 7 dias), sonolência, hipoatividade, palidez, pele infiltrada e hipertelorismo ocular. Não foram estabelecidos critérios objetivos para definição dos sinais clínicos, sendo determinados pela experiência do examinador.

Todos os pacientes foram submetidos, nesse mesmo dia, a coleta de sangue para análise sérica de TSH, 
T4 ou T4 livre e tireoglobulina, radiografia de joelhos para determinação da idade óssea e ultrassonografia de tireoide. Após o resultado dos exames, foi iniciado tratamento com levotiroxina ( 10 a $15 \mu \mathrm{g} / \mathrm{kg} / \mathrm{dia}$ ) para os casos de HCP confirmado.

A análise do TSH em papel-filtro foi realizada por imunofluorimetria por tempo resolvido em um 1235 AutoDelfia $^{\circledR}$ (Automatic Immunoassay System), empregando-se kit AutoDelfia ${ }^{\circledR}$ neonatal hTSH (valores normais $<10 \mathrm{mUI} / \mathrm{L}$ ) e a do TSH, tireoglobulina e T4L pelo método de quimioluminescência (valores normais: 0,3 a $5,0 \mu \mathrm{UI} / \mathrm{mL}, 2$ a $35 \mathrm{ng} / \mathrm{ml} \mathrm{e} 0,8$ a 1,8 $\mathrm{ng} / \mathrm{dL}$, respectivamente). A dosagem de tireoglobulina acima de $200 \mathrm{ng} / \mathrm{ml}$ foi considerada elevada.

As radiografias de joelhos para idade óssea foram realizadas no Serviço de Radiologia do HIJG, sendo utilizado aparelho Siemens ${ }^{\circledR}$ THA BI 150/30/51-100. O médico endocrinologista avaliava as radiografias, observava a presença e tamanho dos núcleos de ossificação dos joelhos (núcleo epifisário distal do fềmur) e estimava a idade óssea. As idades ósseas foram classificadas como normal se os núcleos de ossificação estivessem presentes bilateralmente e maiores que $3 \mathrm{~mm}$, ou como atrasada se um pequeno núcleo $(<3 \mathrm{~mm})$ estivesse presente ou se os núcleos estivessem ausentes.

As ultrassonografias de tireoide foram realizadas no Serviço de Radiologia do HIJG, sendo utilizado aparelho de ultrassonografia Philips $^{\circledR}$ com transdutor multifrequencial de 7,5 a $10 \mathrm{Mhz}$. As conclusões do exame foram classificadas em: tireoide tópica normal, ausência de tecido tireoidiano, glândula ectópica, tireoide aumentada de volume ou tireoide reduzida de tamanho, conforme cálculo do volume glandular. Este era realizado conforme descrito na literatura e de acordo com idade e comprimento da criança $(15,16)$.

Todos os exames eram realizados no mesmo dia da consulta, e os resultados do TSH, T4 total ou livre, ultrassonografia de tireoide, radiografia da idade óssea eram obtidos no mesmo dia da consulta. O resultado da tireoglobulina era observado na consulta de retorno, momento em que se concluía o diagnóstico etiológico, conforme figuras 1 e 2 , e se formaram dois grupos, um constituído pelas disgenesias e o outro por aqueles com tireoide tópica normal ou com bócio. Os pacientes com tireoide tópica normal ou bócio e tireoglobulina normal ou aumentada ficaram com diagnóstico possível de hipotireoidismo transitório ou disormoniogênese, e a definição etiológica somente será concluída após os 3 anos de idade. Foi considerado para o diagnóstico de disormoniogênese apenas aquele paciente com tireoide tópica normal ou bócio e com tireoglobulina baixa devido à etiologia de deficiência de tireoglobulina. Dessa forma, cintilografia ou teste do perclorato não são necessários (17).

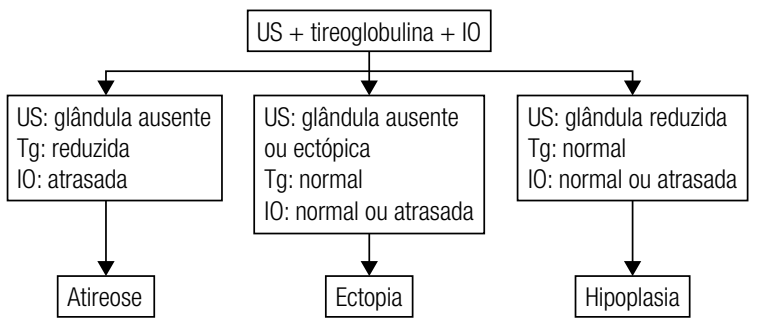

Figura 1. Diagnóstico etiológico de disgenesia tireoidiana*

IO: idade óssea; Tg: dosagem de tireoglobulina; US: ultrassonografia de tireoide. * Modificada de: Gruters A. Angeborene hypothyreose. 2005(9).

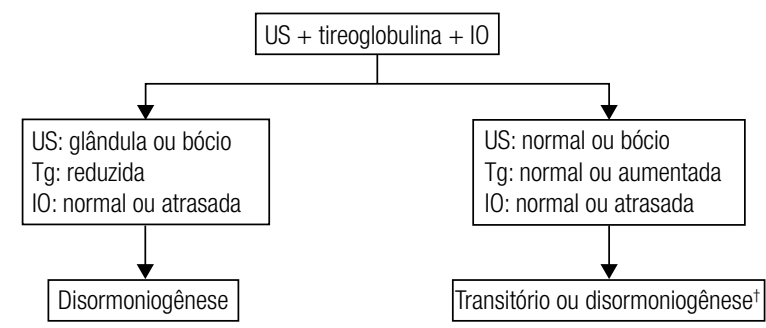

Figura 2. Diagnóstico etiológico com tireoide tópica normal ou bócio*. IO: idade óssea; Tg: dosagem de tireoglobulina; US: ultrassonografia de tireoide. * Modificada de: Gruters A. Angeborene hypothyreose. 2005(9).

${ }^{\dagger}$ Diagnóstico será feito com 3 anos de idade.

O estudo foi previamente aprovado pelo Comitê de Ética e Pesquisa em Seres Humanos do Hospital Infantil Joana de Gusmão sob número 055/2008 e parecer número 070/2008.

A análise estatística foi realizada pelo programa Sestatnet 1999. Para verificar associação entre as variáveis qualitativas nominais, foi utilizado o teste qui-quadrado de Fisher. Entre as variáveis quantitativas sem distribuição normal, foi utilizado o teste U de Mann-Whitney e, para aquelas com distribuição normal, utilizou-se o teste $\mathrm{T}$ de Student. O nível de significância foi de $5 \%$ $(\mathrm{p}<0,05)$.

\section{RESULTADOS}

Entre julho de 2007 e junho de 2009 foram triadas 148.717 crianças pelo PTN-SC, com uma cobertura de $89 \%$ dos nascidos vivos no estado. Das crianças triadas, 63 foram selecionadas para consulta médica e, dessas, 49 apresentaram hipotireoidismo congênito confirma- 
do. Foram selecionados 45 casos para análise, pois os outros casos se mudaram para outro estado ou foram excluídos por ausência de dados.

Das 45 crianças analisadas, $30(66,7 \%)$ eram do sexo feminino e se estabeleceu uma relação de dois femininos para um masculino afetado. A mediana de idade na coleta da triagem neonatal foi cinco dias. A mediana de idade na primeira consulta para aqueles que realizaram somente uma coleta de triagem foi 15 dias e, para os que realizaram duas dosagens, foi 20 dias de vida.

Estabeleceu-se o diagnóstico etiológico em 24 crianças $(53,3 \%)$. Em 51,1\% dos casos o diagnóstico foi disgenesia tireoidiana. A frequência de hipoplasia foi $20 \%$, ectopia $17,8 \%$ e atireose $13,3 \%$. Em relação ao grupo de tireoide tópica normal ou bócio, apenas uma criança $(2,2 \%)$ ficou com diagnóstico estabelecido de disormoniogênese. Em 46,7\% não foi possível diferenciar entre HC transitório ou disormoniogênese e o diagnóstico será concluído após o terceiro ano de vida.

Em relação à avaliação da idade óssea, 12 crianças $(26,6 \%)$ apresentavam idade óssea atrasada em relação à idade cronológica, sendo que, dessas, $80 \%$ tiveram o diagnóstico de atireose. Na ultrassonografia de tireoide, $18(40 \%)$ crianças apresentavam tireoide tópica de volume normal, $13(28,8 \%)$ apresentaram ausência de tecido tireoidiano, $9(20 \%)$ demonstraram redução do volume da glândula, $4(8,8 \%)$ obtiveram bócio como laudo e, em uma criança $(2,2 \%)$, a tireoide foi visualizada ectópica.

Na primeira consulta, em relação aos sinais de hipotireoidismo congênito, os mais prevalentes foram hérnia umbilical $(48,8 \%)$, base nasal alargada $(46,6 \%) \mathrm{e}$ icterícia prolongada além de 7 dias de vida $(44,4 \%)$. Em $20 \%$ das crianças, não foi encontrado nenhum sinal ou sintoma de hipotireoidismo congênito.

De acordo com os antecedentes pessoais pesquisados, $37,7 \%$ das crianças possuíam história familiar de doença tireoidiana. Nenhuma criança acometida apresentava mãe com diagnóstico de hipertireoidismo ou possuía irmão com diagnóstico de hipertireoidismo congênito transitório.

Todas as crianças iniciaram o tratamento com levotiroxina e a média da dose inicial foi $12,69 \mu \mathrm{g} / \mathrm{kg} / \mathrm{dia}$.

$\mathrm{O}$ grupo das disgenesias apresentou diferença significativa para via de parto cesária $(\mathrm{p}=0,02 \mathrm{l})$, para idade óssea atrasada $(\mathrm{p}=0,022)$ e nível de TSH neonatal e sérico muito elevados $(\mathrm{p}=0,0003)$ em comparação com o grupo da tireoide tópica normal ou bócio. As variáveis sexo e história familiar de doença tireoidiana não demonstraram diferença estatística (Tabela 1 ).
Tabela 1. Distribuição de sexo, história familiar, via de parto, idade óssea, nível de TSH sérico de acordo com diagnóstico etiológico no período de julho de 2007 a junho de 2009

\begin{tabular}{|c|c|c|c|c|c|}
\hline \multirow[t]{2}{*}{ Variáveis } & \multicolumn{2}{|c|}{$\begin{array}{l}\text { Tireoide tópica } \\
\text { normal ou bócio* }\end{array}$} & \multicolumn{2}{|c|}{ Disgenesia } & \multirow[t]{2}{*}{$p$ valor ${ }^{\dagger}$} \\
\hline & $\mathbf{N}$ & $\%$ & $\mathbf{n}$ & $\%$ & \\
\hline \multicolumn{6}{|l|}{$\operatorname{SexO}^{\dagger}$} \\
\hline Feminino & 13 & 59,12 & 17 & 73,91 & 0,43 \\
\hline Masculino & 9 & 40,92 & 6 & 26,09 & \\
\hline $\mathrm{HF}^{\dagger}$ & 9 & 40,92 & 8 & 34,78 & 0,79 \\
\hline Parto cesária ${ }^{\dagger}$ & 10 & 45,47 & 19 & 82,61 & 0,021 \\
\hline \multicolumn{6}{|l|}{ Idade óssea ${ }^{\dagger}$} \\
\hline Atrasada & 2 & 9,09 & 10 & 43,48 & 0,022 \\
\hline Normal & 20 & 90,01 & 13 & 56,52 & \\
\hline TSH sérico $(\mu \mathrm{Ul} / \mathrm{ml})^{\S}$ & 22 & 45,94 & 23 & 68,09 & 0,00039 \\
\hline
\end{tabular}

* Considerado como possíveis diagnósticos disormoniogênese ou hipotireoidismo transitório. † Teste qui-quadrado de Fisher; ${ }^{\S}$ Teste U de Mann-Whitney; HF: história familiar de doença tireoidiana.

\section{DISCUSSÃO}

O HCP é uma das causas mais comuns de retardo mental prevenível. Os programas de deteç̧ão neonatal possibilitam o diagnóstico e tratamento precoces $(5,10,11)$.

Os programas de triagem neonatal objetivam ter cobertura de $100 \%$ dos nascidos vivos $(6,9-14,18)$. Em 2005, na América Latina (19) a cobertura foi de 49,3\%. No presente estudo observou-se uma cobertura de $89 \%$, o que é compatível com o descrito, entretanto inferior aos países desenvolvidos $(20,21)$. A menor cobertura pode ser justificada pela realização dos testes de triagem em laboratórios privados e pela inexistência de legislação que obrigue a notificação dos exames realizados e alterados.

No presente foi possível estabelecer o diagnóstico etiológico no primeiro atendimento em $53,3 \%$ dos pacientes. Na Escócia (22), a cintilografia com iodo ${ }^{123}$ era utilizada para avaliar a tireoide e apenas $32,5 \%$ dos pacientes tinham a etiologia determinada. A partir de 1999, foi implementado um novo protocolo que utilizava ultrassonografia e cintilografia e, em 76,2\% dos pacientes, a etiologia ficou conhecida na primeira abordagem (22). Estudos com a utilização exclusiva de ultrassonografia para determinação etiológica do HCP e sobre a porcentagem de diagnósticos realizados na primeira consulta não foram encontrados.

Tahirovic e Toromanovic (23) realizaram avaliação etiológica na primeira consulta com ultrassonografia e encontraram $59 \%$ de disgenesias e $41 \%$ de tireoide tópica. Verificamos, na ultrassonografia, $51,11 \%$ de disgenesia, $28,89 \%$ de glândula ausente, tireoide reduzida em $20 \%$, ectopia em $2,22 \%, 8,89 \%$ de bócio e $40 \%$ de tireoide tópica normal. 
Gruters e cols. (24), que utilizam o mesmo protocolo de diagnóstico usado neste estudo, relatam que, observando a ultrassonografia e a tireoglobulina, $18 \%$ tinham ectopia, $17 \%$, hipoplasia e $48 \%$, atireose. No PTN-SES/ SC, de maneira semelhante, $17,78 \%$ tiveram o diagnóstico de ectopia, $20 \%$ de hipoplasia e $13,3 \%$ tinham atireose.

Hanukoglu e cols. (7), durante a análise da etiologia dos casos de HCP no primeiro atendimento utilizando cintilografia com tecnésio, encontraram $32 \%$ de disormoniogênese. A presença de bócio em um neonato com hipotiroidismo primário sugere hipotiroidismo transitório ou um defeito intrínseco na síntese de hormônios da tireoide. Em Santa Catarina, quatro RN tinham bócio. As mães desses pacientes eram saudáveis, sem bócio e sem história de disfunção tiroidiana, e elas não fizeram uso de nenhuma medicação durante a gestação. Portanto, disormoniogênese foi considerada a causa mais provável de hipotiroidismo nesses pacientes; entretanto, destes, somente um paciente $(2,2 \%)$ teve o diagnóstico confirmado na primeira consulta, em que apresentava tireoglobulina indetectável, com bócio na ultrassonografia (deficiência de tireoglobulina). Essa baixa prevalência possivelmente se deve à dificuldade de realizar esse diagnóstico apenas com dassonografia e tireoglobulina. Em 46,6\% o diagnóstico não foi determinado, pois não foi possível diferenciar entre HCP transitório ou disormoniogênese, e a determinação etiológica será realizada após os 3 anos de idade, como preconizado (6). Sendo assim, possivelmente a frequência real dos casos de disormoniogênese será maior.

Tahirovic e Toromanovic (23) demonstraram que a média do TSH sérico nas disgenesias foi $121 \mu \mathrm{UI} / \mathrm{mL}$ e isso não foi diferente estatisticamente do encontrado nos pacientes com tireoide tópica. A média de TSH sérico que encontramos nas disgenesias foi $68,09 \mu \mathrm{UI} /$ $\mathrm{mL}$, com diferença significativa em comparação ao grupo com tireoide tópica ( $\mathrm{p}=0,0003)$.

Van Vliet e cols. (25) e Wasniewska e cols. (26) verificaram que $87 \%$ e $71,9 \%$, respectivamente, dos pacientes com diagnóstico de atireose possuíam idade óssea atrasada. Em Santa Catarina, o grupo das disgenesias teve maior frequência de idade óssea atrasada $(\mathrm{p}$ $=0,02$ ), sendo que $80 \%$ dos que foram diagnosticados com atireose possuíam idade óssea atrasada.

História familiar de doença tireoidiana e diagnóstico etiológico não apresentaram diferença estatística $(\mathrm{p}=$ $0,7)$ entre os portadores de disgenesia e tireoide tópica. Isso se deve, possivelmente, ao grande número de casos esporádicos. No entanto, nos casos de disormoniogê- nese é realizado aconselhamento genético devido à alta probabilidade de recorrência familiar.

Estudos demonstram que nascidos por cesariana possuem TSH da triagem neonatal mais elevado e justificam esse aumento pelo uso de soluções iodadas para desinfecção da pele materna e posterior amamentação com passagem do iodo pelo leite materno (27). Outros autores relatam que o nível de TSH do cordão umbilical não é influenciado pelo uso de iodo e sim pelo tipo de parto, porém ainda não está determinado se isso afetaria o nível de TSH da triagem neonatal (28).

McElduff e cols. (27) observaram que as crianças nascidas de cesariana têm maior chance de ter HCP (referencial TSH $>15 \mu \mathrm{U} / \mathrm{ml}$ ) e, se for considerado TSH $>5 \mu \mathrm{U} / \mathrm{ml}$, essa chance aumenta. No presente estudo, $64,4 \%$ dos pacientes nasceram de parto cesário, o que está de acordo com o descrito. Quando analisamos os pacientes com disgenesia, $82,6 \%$ nasceram de cesariana e isso teve diferença significativa $(\mathrm{p}=0,02)$ ao comparar com os pacientes com tireoide tópica. Esse resultado é de grande importância clínica e pode ser explicado pela maior gravidade do HCP ao nascer nos pacientes com disgenesia. Entretanto, deve-se chamar atenção para a existência de elevada taxa de cesariana, que pode representar um viés.

Sabe-se que o HCP não apresenta um quadro clínico característico, entretanto alguns sinais são encontrados com frequência nos portadores de $\operatorname{HCP}(6,29)$. Neste trabalho, os sinais e sintomas verificados durante a primeira consulta tiveram uma distribuição semelhante ao de Minas Gerais (30). Em Santa Catarina, os sinais mais prevalentes foram hérnia umbilical $(48,89 \%)$, base nasal alargada $(46,67 \%)$ e icterícia prolongada $(44,44 \%)$. Além disso, $20 \%$ dos pacientes não tinham alterações no exame clínico enquanto, em Minas Gerais (30), apenas 7,9\% eram assintomáticos, o que demonstra grande heterogeneidade da apresentação clínica do HCP, enfatizando a necessidade da triagem neonatal para realizar o diagnóstico precoce.

Nos últimos anos ocorreram aperfeiçoamentos no PTN-SES/SC, principalmente a implantação do protocolo de realização do diagnóstico etiológico no primeiro atendimento, o que representou grande avanço no atendimento dos pacientes com HCP. Ratifica-se que a determinação etiológica na primeira avaliação pode facilitar o entendimento dos pais sobre a importância do tratamento, além de possibilitar ajustes da dose da levotiroxina, visto que é estabelecido que os pacientes que possuem atireose devem manter doses mais elevadas da reposição em relação àqueles com ectopia ou disormoniogênese (17). Sabe-se também que o protocolo de 
investigação etiológica no primeiro atendimento está bem estabelecido na literatura internacional (31), porém ainda é abordado como de caráter opcional. Nesse sentido, deve-se incentivar a implementação desse protocolo nos diversos Serviços de Referência em Triagem Neonatal brasileiros para que o conhecimento da epidemiologia nacional, bem como das especificidades locais, possa fornecer dados para aprimorar os cuidados com os pacientes com hipotireoidismo congênito.

É fundamental que o PTN-SES/SC atinja seus objetivos e que ações políticas para criação de uma legislação específica sobre a coleta de exames em laboratórios privados sejam realizadas. Dessa forma, a cobertura populacional divulgada será de todas as crianças e não somente daquelas que realizaram o teste de triagem em serviço público. Com $100 \%$ das crianças triadas, com tempos de coleta, início do tratamento e avaliação etiológica adequados, a abordagem aos pacientes com HCP atingirá suas metas e contribuirá significativamente para prevenção de agravos.

Declaração: os autores declaram não haver conflitos de interesse científico neste estudo.

\section{REFERÊNCIAS}

1. Park SM, Chatterjee VK. Genetics of congenital hypothyroidism. J Med Genet. 2005;42(5):379-89.

2. Bevenides $A M$, Lima $C H$, Rocha $C A$, Corrêa AR, El Husny AS, Fernandes-Caldato MC. Perfil epidemiológico de portadores de hipotireoidismo congênito. Rev Para Med. 2006;20(3):23-6.

3. Castanet M, Polak M, Bonaiti-Pellie C, Lyonnet S, Czernichow $P$, Leger J. Nineteen years of national screening for congenital hypothyroidism: familial cases with thyroid dysgenesis suggest the involvement of genetic factors. J Clin Endocrinol Metab. 2001;86(5):2009-14.

4. Rovet J. Congenital hypothyroidism: treatment and outcome. Curr Opin Endocrinol Diabetes. 2005;12(1):42-52.

5. Nascimento ML, Pires MMS, Nassar SM, Ruhland L. Avaliação do programa de rastreamento neonatal para hipotireoidismo congênito da Secretaria de Estado da Saúde de Santa Catarina. Arq Bras Endocrinol Metab. 2003;47(1):75-81.

6. American Academy of Pediatrics, Section on Endocrinology and Committee on Genetics, American Thyroid Association and Committee on Public Health, Lawson Wilkins Pediatric Endocrine Society. Update of newborn screening and therapy for congenital hypothyroidism. Pediatrics. 2006;117(6):2290-303.

7. Hanukoglu A, Perlman K, Shamis I, Brnjac L, Rovet J, Daneman D. Relationship of etiology to treatment in congenital hypothyroidism. J Clin Endocrinol Metab. 2001;86(1):186-91.

8. LaFranchi S. Congenital hypothyroidism: etiologies, diagnosis, and management. Thyroid. 1999;9(7):735-40.

9. Gruters A. Angeborene Hypothyreose. In: Michalk D, Schönau E. Differentialdiagnose Pädiatrie. 2.ed. Munchen: Elsevier; 2005; p. 508-11.

10. Gruters A. Congenital hypothyroidism. Pediatr Ann. 1992;21(1):15, 8-21, 4-8.

11. Nascimento ML, Silva PC, Simoni G, Lobo GS, Souza CD. [Congenital hypothyroidism screening program: preliminary results]. J Pediatr (Rio J). 1997;73(3):176-9.
12. Rubió I. Novos conhecimentos sobre a etiologia molecular das disgenesias tireoidianas. In: Medeiros-Neto G, Knobel M. Hipotireoidismo congênito no Brasil: desafios à busca de soluções. São Paulo: Conectfarma Publicações Científicas; 2008. p. 19-26.

13. Eugster EA, LeMay D, Zerin JM, Pescovitz $\mathrm{OH}$. Definitive diagnosis in children with congenital hypothyroidism. J Pediatr. 2004;144(5):643-7.

14. Ohnishi H, Sato H, Noda H, Inomata H, Sasaki N. Color Doppler ultrasonography: diagnosis of ectopic thyroid gland in patients with congenital hypothyroidism caused by thyroid dysgenesis. $J$ Clin Endocrinol Metab. 2003;88(11):5145-9.

15. Ueda D, Mitamura R, Suzuki N, Yano K, Okuno A. Sonographic imaging of the thyroid gland in congenital hypothyroidism. Pediatr Radiol. 1992;22:102-5.

16. Perry RJ, Hollman AS, Wood AM, Donaldson MDC. Ultrasound of the thyroid gland in the newborn: normative data. Arch Dis Child Fetal Neonatal Ed. 2002;87:F209-11.

17. Beltrao CB, Juliano AG, Chammas MC, Watanabe T, Sapienza MT, Marui S. Etiology of congenital hypothyroidism using thyroglobulin and ultrasound combination. Endocr J. 2010;57(7):587-93.

18. Gruters A, Krude H. Update on the management of congenital hypothyroidism. Horm Res. 2007;68 Suppl 5:107-11.

19. Borrajo GJ. Newborn screening in Latin America at the beginning of the 21st century. J Inherit Metab Dis. 2007;30(4):466-81.

20. Olivieri A. The Italian National Register of infants with congenital hypothyroidism: twenty years of surveillance and study of congenital hypothyroidism. Riv Ital Pediatr. 2009;35(1):2.

21. Magalhaes PK, Turcato Mde F, Angulo Ide L, Maciel LM. [Neonatal screening program at the university hospital of the Ribeirao Preto School of Medicine, Sao Paulo University, Brazil]. Cad Saude Publica. 2009;25(2):445-54.

22. Jones JH, Mackenzie J, Croft GA, Beaton S, Young D, Donaldson MD. Improvement in screening performance and diagnosis of congenital hypothyroidism in Scotland 1979-2003. Arch Dis Child. 2006;91(8):680-5.

23. Tahirovic $\mathrm{H}$, Toromanovic A. Neonatal screening for congenital hypothyroidism in the Federation of Bosnia and Herzegovina: eight years' experience. Eur J Pediatr. 2009;168(5):629-31.

24. Gruters A, Jenner A, Krude H. Long-term consequences of congenital in the era of screening programmes. Best Pract Res Clin Endocrinol Metab. 2002;16(2):369-82.

25. Van Vliet G, Larroque B, Bubuteishvili L, Supernant K, Leger J. Sex-specific impact of congenital hypothyroidism due to thyroid dysgenesis on skeletal maturation in term newborns. J Clin Endocrinol Metab. 2003;88(5):2009-13.

26. Wasniewska M, De Luca F, Cassio A, Oggiaro N, Gianino P, Delvecchio $\mathrm{M}$, et al. In congenital hypothyroidism bone maturation at birth may be a predictive factor of psychomotor development during the first year of life irrespective of other variables related to treatment. Eur J Endocrinol. 2003;149(1):1-6.

27. McElduff A, McElduff $P$, Wiley $V$, Wilcken B. Neonatal thyrotropin as measured in a congenital hypothyroidism screening program: influence of the mode of delivery. J Clin Endocrinol Metab. 2005;90(12):6361-3.

28. Ordookhani A, Pearce EN, Mirmiran P, Azizi F, Braverman LE. The effect of type of delivery and povidone-iodine application at delivery on cord dried-blood-specimen thyrotropin level and the rate of hyperthyrotropinemia in mature and normal-birth-weight neonates residing in an iodine-replete area: report of Tehran Province, 1998-2005. Thyroid. 2007;17(11):1097-102.

29. Nascimento ML. Hipotireoidismo congênito em Santa Catarina. In: Medeiros-Neto G, Knobel M. Hipotireoidismo congênito no Brasil: desafios à busca de soluções. São Paulo: Conectfarma Publicações Científicas 2008;11:67-70.

30. Pezzuti IL, de Lima PP, Dias VM. Congenital hypothyroidism: the clinical profile of affected newborns identified by the Newborn Screening Program of the State of Minas Gerais, Brazil. J Pediatr (Rio J). 2009;85(1):72-9.

31. La Franchi SH. Approach to the diagnosis and treatment of neonatal hypothyroidism. J Clin Endocrinol Metab. 2011;96(10):2959-67. 